\title{
Construction of orthonormal wavelet-like bases
}

\author{
Patricia Mariela Morillas ${ }^{\text {a) }}$ \\ Instituto de Matemática Aplicada San Luis, UNSL-CONICET, Ejército de los Andes 950, \\ 5700 San Luis, Argentina
}

(Received 18 March 2010; accepted 17 June 2010; published online 12 August 2010)

A general method for constructing wavelet-like bases in a Hilbert space $\mathcal{H}$ starting from any orthonormal basis in $\mathcal{H}$ and any periodic orthonormal wavelet basis is presented. With this method we can take advantage of the characteristics of both types of bases to obtain orthonormal wavelet-like bases that are suitable to represent functions and operators efficiently. (c) 2010 American Institute of Physics. [doi:10.1063/1.3462714]

\section{INTRODUCTION}

The applicability of wavelets in different areas of pure and applied mathematics depends on their ability to represent a wide class of functions and operators efficiently (for a treatment of the theory of wavelets see, e.g., Refs. 3, 10, 13, 14, and 16). In particular, this fact is useful in the development of fast adaptive numerical algorithms (see, e.g., Refs. 1, 2, 5, and 6). As mentioned in Ref. 15 (see also Ref. 11), wavelets are applied to the study of problems in different physical fields such as astrophysics, turbulence, meteorology, plasma physics, atomic and solid state physics, multifractals occurring in physics, biophysics (in medicine and physiology), and computational and mathematical physics.

New examples of wavelets with good properties are always required. One important property is that functions and operators should have representations in the wavelet basis with only a small number of significant coefficients, i.e., effective sparse representations. If wavelet bases are associated with a multiresolution analysis, we can change the level of the approximation of functions and operators using wavelet transforms. Smooth wavelets are important to represent differential operators. Explicit expressions of wavelets are useful for performing computations.

Based on the ideas introduced in Refs. 7 and 9 but using a different approach, we present in this paper a general method for constructing wavelet-like bases in a Hilbert space $\mathcal{H}$, associated with multiresolution analysis, starting from any orthonormal basis in $\mathcal{H}$ and any periodic orthonormal wavelet basis. With this method we can take advantage of the characteristics of both types of bases to obtain orthonormal wavelet-like bases that are suitable to represent functions and operators efficiently. The method admits variants, and, in particular, we can obtain smoothness, explicit expressions, and effectively sparse representations for the wavelet-like functions.

The construction method is the combination of two procedures for obtaining orthonormal wavelet-like bases. In one of them, we use any given unitary operator and any given periodic orthonormal wavelet basis. This procedure is intended to lead to orthonormal wavelet-like bases that permit effectively sparse representations. The second procedure uses any given unitary matrix and any given orthonormal basis in $\mathcal{H}$ and permits to obtain smoothness and explicit expressions. We then combine these two procedures for constructing smooth wavelet-like functions with explicit expressions that permit effectively sparse representations.

We illustrate the method by obtaining orthonormal wavelet-like bases based on trigonometric functions and eigenfunctions of Schrödinger and Chebyshev differential operators. We show that

\footnotetext{
${ }^{a)}$ Electronic mail: morillas@unsl.edu.ar.
} 
using eigenfunctions of an operator $L$, if $S$ is certain perturbation of $L$, then the stiffness matrix in the wavelet-Galerkin scheme for differential equations associated with $S$ can be preconditioned by a diagonal matrix so that the condition number is uniformly bounded.

The rest of this paper is organized as follows. In Sec. II we introduce the concepts of multiresolution analysis, scaling-like and wavelet-like vectors in a separable Hilbert space. We also establish some basic results. In Sec. III we present the general method for constructing waveletlike bases. Then, in Sec. IV, some examples of wavelet-like bases based on trigonometric functions and eigenfunctions of differential operators are presented. Finally, some final remarks are included in Sec. V.

\section{WAVELET-LIKE ORTHONORMAL BASES IN A HILBERT SPACE}

In this section we introduce some basic concepts and results that will be use in the rest of the paper. Let $\mathcal{H}$ be a separable Hilbert space and let $j_{0} \geq 0$.

Definition 2.1: The sequence $\left\{V_{j}: j \geq j_{0}\right\}$ is a multiresolution analysis (MRA) of $\mathcal{H}$ if

(1) $V_{j}$ is a subspace of $\mathcal{H}, j \geq j_{0}$;

(2) the dimension of $V_{j}$ is $2^{j}, j \geq j_{0}$;

(3) $V_{j} \subset V_{j+1}, j \geq j_{0}$;

(4) $\cup_{j=j_{0}}^{\infty} V_{j}$ is dense in $\mathcal{H}$.

Theorem 2.2: If $\left\{V_{j}: j \geq j_{0}\right\}$ is a MRA of $\mathcal{H}, W_{j}=V_{j+1} \cap V_{j}^{\perp}=V_{j+1} \ominus V_{j}$ and $j_{1} \geq j_{0}$, then $\mathcal{H}$ $=V_{j_{1}} \oplus\left(\oplus_{j=j_{1}}^{\infty} W_{j}\right)$.

Definition 2.3: If

(1) $\left\{\Phi_{j, k}\right\}_{k=0}^{2^{j}-1}, j \geq j_{0}$, is an orthonormal system in $\mathcal{H}$;

(2) the sequence $V_{j}=\operatorname{span}\left\{\Phi_{j, k}\right\}_{k=0}^{2^{j}-1}, j \geq j_{0}$, is a MRA of $\mathcal{H}$;

then $\cup_{j=j_{0}}^{\infty}\left\{\Phi_{j, k}\right\}_{k=0}^{j^{j}-1}$ is called a scaling-like system in $\mathcal{H}$ and each $\Phi_{j, k}$ is called a scaling-like vector.

Definition 2.4: If

(1) $\cup_{j=j_{0}}^{\infty}\left\{\Psi_{j, k}\right\}_{k=0}^{2^{j}-1}$ is an orthonormal system in $\mathcal{H}$;

(2) there exists a scaling-like system $\cup_{j=j_{0}}^{\infty}\left\{\Phi_{j, k}\right\}_{k=0}^{j}-1$ for $\mathcal{H}$, such that for all $j \geq j_{0}, k, k^{\prime}$ $=0, \ldots, 2^{j}-1,\left\langle\Phi_{j, k}, \Psi_{j, k^{\prime}}\right\rangle_{\mathcal{H}}=0$;

then $\cup_{j=j_{0}}^{\infty}\left\{\Psi_{j, k}\right\}_{k=0}^{j^{j}-1}$ is called a wavelet-like system for $\mathcal{H}$, associated with $\cup_{j=j_{0}}^{\infty}\left\{\Phi_{j, k}\right\}_{k=0}^{2^{j}-1}$, and each $\Psi_{j, k}$ is called a wavelet-like vector.

Theorem 2.5: If $\cup_{j=j_{0}}^{\infty}\left\{\Phi_{j, k}\right\}_{k=0}^{2^{j}-1}$ is a scaling-like system for $\mathcal{H}$ with a wavelet-like system $\cup_{j=j_{0}}^{\infty}\left\{\Psi_{j, k}\right\}_{k=0}^{2^{j}-1}$ and $W_{j}$ is as in Theorem 2.2, then $W_{j}=\operatorname{span}\left\{\Psi_{j, k}\right\}_{k=0}^{2^{j}-1}$.

From Theorem 2.2 and Theorem 2.5, we obtain the following.

Theorem 2.6: If $\cup_{j=j_{0}}^{\infty}\left\{\Phi_{j, k}\right\}_{k=0}^{2^{j}-1}$ is a scaling-like system for $\mathcal{H}$ with a wavelet-like system $\cup_{j=j_{0}}^{\infty}\left\{\Phi_{j, k}\right\}_{k=0}^{j_{-1}-1}$ and $j_{1} \geq j_{0}$, then $\left\{\Phi_{j_{1}, k}\right\}_{k=0}^{2_{1} j_{1}-1} \cup \cup_{j=j_{1}}^{\infty}\left\{\Psi_{j, k}\right\}_{k=0}^{j_{-1}-1}$ is an orthonormal basis of $\mathcal{H}$.

We will call the basis of Theorem 2.6 a wavelet-like basis for $\mathcal{H}$.

Remark 2.7: As a consequence of the finite dimensionality, each MRA of $\mathcal{H},\left\{V_{j}: j \geq j_{0}\right\}$, has an associated scaling-like system $\cup_{j=j_{0}}^{\infty}\left\{\Phi_{j, k}\right\}_{k=0}^{2^{j}-1}$, such that $V_{j}=\operatorname{span}\left\{\Phi_{j, k}\right\}_{k=0}^{2^{j}-1}, j \geq j_{0}$. Also, for each scaling-like system for $\mathcal{H}$, there exists an associated wavelet-like system.

In the sequel, $\left(p_{j}\right)$ denotes the column vector with components $p_{j, k}, k=0, \ldots, 2^{j}-1$, where each $p_{j, k}$ is an element of a certain class. For example, $\left(\Phi_{j}\right)$ denotes the column vector with components $\Phi_{j, k}, k=0, \ldots, 2^{j}-1$, and if $f \in \mathcal{H}$, then $\left(\left\langle f, \Phi_{j}\right\rangle_{\mathcal{H}}\right)$ denotes the column vector with components $\left\langle f, \Phi_{j, k}\right\rangle_{\mathcal{H}}, k=0, \ldots, 2^{j}-1$.

Definition 2.8: Let $j \geq 1$. If $H_{j}, G_{j}$ are matrices of order $2^{j-1} \times 2^{j}$, such that $H_{j} H_{j}^{*}=G_{j} G_{j}^{*}=I$, $H_{j} G_{j}^{*}=G_{j} H_{j}^{*}=O$, and $H_{j}^{*} H_{j}+G_{j}^{*} G_{j}=I$, then $\left(H_{j}, G_{j}\right)$ will be called a filter pair. 
Theorem 2.9: $\cup_{j=j_{0}}^{\infty}\left\{\Phi_{j, k}\right\}_{k=0}^{2^{j}-1}$ is a scaling-like system for $\mathcal{H}$ with a wavelet-like system $\cup_{j=j_{0}}^{\infty}\left\{\Psi_{j, k}\right\}_{k=0}^{j^{j}-1}$, if and only if there exists $j_{1} \geq j_{0}$, such that $\left\{\Phi_{j_{1}, k}\right\}_{k=0}^{j_{1}-1} \cup \cup_{j=j_{1}}^{\infty}\left\{\Psi_{j, k}\right\}_{k=0}^{j^{j}-1}$ is an orthonormal basis of $\mathcal{H}$ and there exist (unique) filter pairs, $\left(H_{j}, G_{j}\right), j \geq j_{0}+1$, such that for $j \geq j_{0}+1$,

$$
\left(\Phi_{j-1}\right)=H_{j}\left(\Phi_{j}\right), \quad\left(\Psi_{j-1}\right)=G_{j}\left(\Phi_{j}\right),\left(\Phi_{j}\right)=H_{j}^{*}\left(\Phi_{j-1}\right)+G_{j}^{*}\left(\Psi_{j-1}\right) .
$$

Proof: Suppose $\cup_{j=j_{0}}^{\infty}\left\{\Phi_{j, k}\right\}_{k=0}^{2^{j}-1}$ is a scaling-like system for $\mathcal{H}$ with a wavelet-like system $\cup_{j=j_{0}}^{\infty}\left\{\Psi_{j, k}\right\}_{k=0}^{j-1}$. Let $H_{j}, G_{j}, j \geq j_{0}+1$, be the matrices of order $2^{j-1} \times 2^{j}$, with entries $\left\langle\Phi_{j-1, k}, \Phi_{j, k^{\prime}}\right\rangle_{\mathcal{H}}$ and $\left\langle\Psi_{j-1, k}, \Phi_{j, k^{\prime}}\right\rangle_{\mathcal{H}}, k=0, \ldots, 2^{j-1}-1, k^{\prime}=0, \ldots, 2^{j}-1$, respectively. Since $V_{j}, W_{j} \subset V_{j+1}, V_{j+1}=V_{j}$ $\oplus W_{j},\left\{\Phi_{j, k}\right\}_{k=0}^{2^{j}-1}$ is an orthonormal basis of $V_{j}$ and $\left\{\Psi_{j, k}\right\}_{k=0}^{2^{j}-1}$ is an orthonormal basis of $W_{j}, j \geq j_{0}$, it is easy to see that $(1)$ holds and $\left(H_{j}, G_{j}\right)$ are filter pairs. Moreover, by Theorem 2.6, for all $j_{1}$ $\geq j_{0},\left\{\Phi_{j_{1}, k}\right\}_{k=0}^{j_{1}^{j_{1}}-1} \cup \cup_{j=j_{1}}^{\infty}\left\{\Psi_{j, k}\right\}_{k=0}^{2^{j}-1}$ is an orthonormal basis of $\mathcal{H}$.

Suppose now that there exists $j_{1} \geq j_{0}$, such that $\left\{\Phi_{j_{1}, k}\right\}_{k=0}^{j_{1}{ }_{1}} \cup \cup_{j=j_{1}}^{\infty}\left\{\Psi_{j, k}\right\}_{k=0}^{j^{j}-1}$ is an orthonormal basis of $\mathcal{H}$ and there exist filter pairs, $\left(H_{j}, G_{j}\right), j \geq j_{0}+1$, such that (1) holds. Let $V_{j}$ $=\operatorname{span}\left\{\Phi_{j, k}\right\}_{k=0}^{2^{j}-1}, W_{j}=\operatorname{span}\left\{\Psi_{j, k}\right\}_{k=0}^{2^{j}-1}, j \geq j_{0}$. Since $\left\{\Phi_{j_{1}, k}\right\}_{k=0}^{j_{1} j_{1}} \cup \cup_{j=j_{1}}^{\infty}\left\{\Psi_{j, k}\right\}_{k=0}^{2^{j}-1}$ is an orthonormal system in $\mathcal{H}$ and $\left(H_{j}, G_{j}\right)$ are filter pairs, by (1), $\cup_{j=j_{0}}^{\infty}\left\{\Phi_{j, k}\right\}_{k=0}^{2^{j}-1} \cup \cup_{j=j_{0}}^{\infty}\left\{\Psi_{j, k}\right\}_{k=0}^{j^{j}-1}$ is an orthonormal system in $\mathcal{H}, H_{j}, G_{j}, j \geq j_{0}+1$, are the matrices with entries $\left\langle\Phi_{j-1, k}, \Phi_{j, k^{\prime}}\right\rangle_{\mathcal{H}}$ and $\left\langle\Psi_{j-1, k}, \Phi_{j, k^{\prime}}\right\rangle_{\mathcal{H}}$, $k=0, \ldots, 2^{j-1}-1, \quad k^{\prime}=0, \ldots, 2^{j}-1, \quad$ respectively, and $V_{j}, W_{j} \subset V_{j+1}, \quad V_{j+1}=V_{j} \oplus W_{j}$. Since $\left\{\Phi_{j_{1}, k}\right\}_{k=0}^{2^{j}{ }_{1-1}} \cup \cup_{j=j_{1}}^{\infty}\left\{\Psi_{j, k}\right\}_{k=0}^{2^{j}-1}$ is an orthonormal basis of $\mathcal{H}$, then $\mathcal{H}=V_{j_{1}} \oplus\left(\oplus_{j=j_{1}}^{\infty} W_{j}\right)$ and thus $\cup_{j=j_{0}}^{\infty} V_{j}$ is dense in $\mathcal{H}$. We conclude that $\cup_{j=j_{0}}^{\infty}\left\{\Phi_{j, k}\right\}_{k=0}^{2^{j}-1}$ is a scaling-like system for $\mathcal{H}$ and $\cup_{j=j_{0}}^{\infty}\left\{\Psi_{j, k}\right\}_{k=0}^{j}$ is an associated wavelet-like system.

In view of Theorem 2.9 we say that $\left(H_{j}, G_{j}\right), j \geq j_{0}+1$, are the filter pairs of $\cup_{j=j_{0}}^{\infty}\left\{\Phi_{j, k}\right\}_{k=0}^{j}$ and $\cup_{j=j_{0}}^{\infty}\left\{\Psi_{j, k}\right\}_{k=0}^{j^{j}-1} . H_{j}$ and $G_{j}$ are the matrices with entries $\left\langle\Phi_{j-1, k}, \Phi_{j, k^{\prime}}\right\rangle_{\mathcal{H}}$ and $\left\langle\Psi_{j-1, k}, \Phi_{j, k^{\prime}}\right\rangle_{\mathcal{H}}, k$ $=0, \ldots, 2^{j-1}-1, k^{\prime}=0, \ldots, 2^{j}-1$, respectively.

As a consequence of Theorem 2.9, we obtain the following.

Theorem 2.10: If $\cup_{j=j_{0}}^{\infty}\left\{\Phi_{j, k}\right\}_{k=0}^{2^{j}-1}$ is a scaling-like system for $\mathcal{H}$ with a wavelet-like system $\cup_{j=j_{0}}^{\infty}\left\{\Phi_{j, k}\right\}_{k=0}^{j}$, filter pairs $\left(H_{j}, G_{j}\right), j \geq j_{0}+1$, and $f \in \mathcal{H}$, then

$$
\begin{gathered}
\left(\left\langle f, \Phi_{j-1}\right\rangle_{\mathcal{H}}\right)=H_{j}\left(\left\langle f, \Phi_{j}\right\rangle_{\mathcal{H}}\right),\left(\left\langle f, \Psi_{j-1}\right\rangle_{\mathcal{H}}\right)=G_{j}\left(\left\langle f, \Phi_{j}\right\rangle_{\mathcal{H}}\right), \\
\left(\left\langle f, \Phi_{j}\right\rangle_{\mathcal{H}}\right)=H_{j}^{*}\left(\left\langle f, \Phi_{j-1}\right\rangle_{\mathcal{H}}\right)+G_{j}^{*}\left(\left\langle f, \Psi_{j-1}\right\rangle_{\mathcal{H}}\right) .
\end{gathered}
$$

Although the concepts of MRA, scaling-like and wavelet-like vectors, and their properties are simple, to exhibit concrete interest examples for particular Hilbert spaces $\mathcal{H}$ can be a difficult task. The most studied case is $\mathcal{H}=L^{2}(T)$. The simplest way to construct a MRA for $L^{2}(T)$ is the periodization (see, e.g., Refs. 10, 13, and 16). Let $\phi, \psi \in L^{2}(\mathbb{R}) \cap L^{1}(\mathbb{R})$ be a scaling function and a wavelet, respectively, of a multiresolution analysis on the real line. Let $\phi_{j, k}^{\text {per }}, \psi_{j, k}^{\text {per }}, j \geq 0$, be the functions obtained by the periodization of the scaling functions $\phi_{j, k}$ and the wavelets $\psi_{j, k}$, respectively, i.e.,

$$
\phi_{j, k}^{p e r}=\sum_{l \in \mathbb{Z}} \phi_{j, k}(x+l)=2^{j / 2} \sum_{l \in \mathbb{Z}} \phi\left(2^{j}(x+l)-k\right)
$$

and

$$
\psi_{j, k}^{p e r}=\sum_{l \in \mathbb{Z}} \psi_{j, k}(x+l)=2^{j / 2} \sum_{l \in \mathbb{Z}} \psi\left(2^{j}(x+l)-k\right) .
$$

In the sequel we suppose that both $\phi, \psi$ have radial decreasing $L^{1}(\mathbb{R})$-majorants [for a definition of radial decreasing $L^{1}(\mathbb{R})$-majorant see (3.1) of Chap. 5 in Ref. 13]. In this case $\cup_{j=j_{0}}^{\infty}\left\{\phi_{j, k}^{p e r}\right\}_{k=0}^{2^{j}-1}$ is a 
scaling-like system for $L^{2}(T)$ with an associated wavelet-like system $\cup_{j=j_{0}}^{\infty}\left\{\psi_{j, k}^{p e r}\right\}_{k=0}^{2^{j}-1}$ (see, e.g., Ref. 13).

Lemma 2.11: Suppose that $\phi_{j, k}=\Sigma_{m \in \mathbb{Z}} a_{k, m}^{j+1} \phi_{j+1, m}$ and $\psi_{j, k}=\Sigma_{m \in Z} b_{k, m}^{j+1} \phi_{j+1, m}$. If $H_{j}^{p e r}, G_{j}^{p e r}$ are the matrices of order $2^{j-1} \times 2^{j}, \quad j>0$, with entries $H_{j}^{\text {per }}(k, m)=\Sigma_{s \in Z^{\prime}} a_{k, m+2^{j} s}$ and $G_{j}^{\text {per }}(k, m)$ $=\sum_{s \in Z} b_{k, m+2 j_{s}}^{j}$, then

$$
\begin{gathered}
\left(\phi_{j-1}^{\text {per }}\right)=H_{j}^{\text {per }}\left(\phi_{j}^{\text {per }}\right), \\
\left(\psi_{j-1}^{\text {per }}\right)=G_{j}^{\text {per }}\left(\phi_{j}^{\text {per }}\right),\left(\phi_{j}^{\text {per }}\right)=\left(H_{j}^{\text {per }}\right)^{*}\left(\phi_{j-1}^{\text {per }}\right)+\left(G_{j}^{\text {per }}\right)^{*}\left(\psi_{j-1}^{\text {per }}\right) .
\end{gathered}
$$

As a consequence of the above lemma, $\left(H_{j}^{p e r}, G_{j}^{p e r}\right), j>0$, are the filter pairs of $\cup_{j=j}^{\infty}\left\{\phi_{j, k}^{p e r}\right\}_{k=0}^{j_{-}-1}$ and $\cup_{j=j_{0}}^{\infty}\left\{\psi_{j, k}^{p e r}\right\}_{k=0}^{2^{j}-1}$.

\section{CONSTRUCTION OF WAVELET-LIKE ORTHONORMAL BASES}

In this section we describe procedures to obtain a MRA of $\mathcal{H}$.

\section{A. Construction of a MRA of $\mathcal{H}$ starting from a MRA of $L^{2}(T)$ and a unitary operator $U: L^{2}(T) \rightarrow \mathcal{H}$}

Theorem 3.1: $\cup_{j=j_{0}}^{\infty}\left\{\Phi_{j, k}\right\}_{k=0}^{2^{j}-1}$ is a scaling-like system for $\mathcal{H}$ with a wavelet-like system $\cup_{j=j_{0}}^{\infty}\left\{\Psi_{j, k}\right\}_{k=0}^{2^{j}-1}$ and filter pairs $\left(H_{j}, G_{j}\right)=\left(H_{j}^{p e r}, G_{j}^{p e r}\right)$ if and only if there exists a (unique) unitary operator $U: L^{2}(T) \rightarrow \mathcal{H}$, such that

$$
\Phi_{j, k}=U \phi_{j, k}^{p e r}
$$

and

$$
\Psi_{j, k}=U \psi_{j, k}^{p e r} .
$$

Proof: Suppose that $U: L^{2}(\mathbb{T}) \rightarrow \mathcal{H}$ is a unitary operator such that $\Phi_{j, k}=U \phi_{j, k}^{p e r}$ and $\Psi_{j, k}$ $=U \psi_{j, k}^{p e r}$.

Since $U$ preserves inner products, $\left\{\phi_{j, k}^{p e r}\right\}_{k=0}^{2^{j}-1}, j \geq j_{0}$, and $\cup_{j=j_{0}}^{\infty}\left\{\psi_{j, k}^{p e r}\right\}_{k=0}^{2^{j}-1}$ are orthonormal systems in $L^{2}(T)$, then $\left\{\Phi_{j, k}\right\}_{k=0}^{2^{j}-1}, j \geq j_{0}$, and $\cup_{j=j_{0}}^{\infty}\left\{\Psi_{j, k}\right\}_{k=0}^{j}-1$ are orthonormal systems in $\mathcal{H}$. Therefore, $\left\{\Phi_{j, k}\right\}_{k=0}^{2^{j}-1}$ is linearly independent and thus the dimension of $V_{j}$ is $2^{j}$.

We also have $V_{j}=U V_{j}^{\text {per }}$ and $W_{j}=U W_{j}^{\text {per }}$. Then $V_{j}=U V_{j}^{\text {per }} \subset U V_{j+1}^{\text {per }}=V_{j+1}$.

Let $g \in \mathcal{H}$ and $\epsilon>0$. Since $U$ is onto, there exists $f \in L^{2}(T)$, such that $g=U f$. Since $\cup_{j=j_{0}} V_{j}^{\text {per }}$ is dense in $L^{2}(\mathbb{T})$, there exists $f_{\epsilon} \in \cup_{j=j_{0}} V_{j}^{\text {per }}$, such that $\left\|f-f_{\epsilon}\right\|_{L^{2}(\mathbb{T})}<\epsilon$. Suppose $f_{\epsilon} \in V_{j}^{\text {per }}$. Then $U f_{\epsilon} \in V_{j}$ and $\left\|g-U f_{\epsilon}\right\|_{\mathcal{H}}=\left\|U f-U f_{\epsilon}\right\|_{\mathcal{H}}=\left\|f-f_{\epsilon}\right\|_{L^{2}(\mathrm{~T})}<\epsilon$. This shows that $\cup_{j=j_{0}} V_{j}$ is dense in $\mathcal{H}$.

Thus we have proven that $\cup_{j=j_{0}}^{\infty}\left\{\Phi_{j, k}\right\}_{k=0}^{2^{j}-1}$ is a scaling-like system for $\mathcal{H}$.

Since $U$ preserves inner products, we also have for $j \geq j_{0}, k, k^{\prime}=0, \ldots, 2^{j}-1,\left\langle\Phi_{j, k}, \Psi_{j, k^{\prime}}\right\rangle_{\mathcal{H}}$ $=\left\langle\phi_{j, k}^{\text {per }}, \psi_{j, k^{\prime}}^{\text {per }}\right\rangle_{L^{2}(\mathrm{~T})}=0$. Then $\cup_{j=j_{0}}^{\infty}\left\{\Psi_{j, k}\right\}_{k=0}^{2^{j}-1}$ is an associated wavelet-like system.

Finally, for $k=0, \ldots, 2^{j-1}-1$ and $k^{\prime}=0, \ldots, 2^{j}-1,\left\langle\Phi_{j-1, k}, \Phi_{j, k^{\prime}}\right\rangle_{\mathcal{H}}=\left\langle\phi_{j, k}^{p e r}, \phi_{j-1, k^{\prime}}^{p e r}\right\rangle_{L^{2}(\mathrm{~T})}$ and $\left\langle\Psi_{j-1, k}, \Phi_{j, k^{\prime}}\right\rangle_{\mathcal{H}}=\left\langle\psi_{j, k}^{\text {per }}, \phi_{j-1, k^{\prime}}^{\text {per }}\right\rangle_{L^{2}(\mathbb{T})}$. Then, $H_{j}=H_{j}^{\text {per }}$ and $G_{j}=G_{j}^{\text {per }}$.

Suppose now that $\cup_{j=j_{0}}^{\infty}\left\{\Phi_{j, k}\right\}_{k=0}^{2^{j}-1}$ is a scaling-like system for $\mathcal{H}$ with a wavelet-like system $\cup_{j=j_{0}}^{\infty}\left\{\Psi_{j, k}\right\}_{k=0}^{j_{-}-1}$ and filter pairs $\left(H_{j}, G_{j}\right)=\left(H_{j}^{\text {per }}, G_{j}^{\text {per }}\right)$. For $j_{1} \geq j_{0}$ consider the unique unitary operator $U: L^{2}(\mathbb{T}) \rightarrow \mathcal{H}$, such that $\Phi_{j_{1}, k}=U \phi_{j_{1}, k}^{\text {per }}$ and $\Psi_{j, k}=U \psi_{j, k}^{\text {per }}, j \geq j_{1}$. We have 


$$
\Phi_{j_{1}-1, k}=\sum_{l=0}^{2^{j_{1}-1}} H_{j_{1}}(k, l) \Phi_{j_{1}, l}=\sum_{l=0}^{2^{j_{1}-1}} H_{j_{1}}^{\text {per }}(k, l) U \phi_{j_{1}, l}^{\text {per }}=U \phi_{j_{1}-1, k}^{\text {per }} .
$$

Following in this manner it can be proven that for $j<j_{1}, \Phi_{j, k}=U \phi_{j, k}^{p e r}$. Similarly, $\Psi_{j, k}=U \psi_{j, k}^{\text {per }}$ for $j<j_{1}$. We now have

$$
\begin{aligned}
\Phi_{j_{1}+1, k}= & \sum_{l=0}^{2^{j_{1}-1}} H_{j_{1}+1}^{*}(k, l) \Phi_{j_{1}, l}+\sum_{l=0}^{2^{j_{1}-1}} G_{j_{1}+1}^{*}(k, l) \Psi_{j_{1}, l}=\sum_{l=0}^{2^{j_{1}-1}}\left(H_{j_{1}+1}^{\text {per }}\right)^{*}(k, l) U \phi_{j_{1}, l}^{\text {per }} \\
& +\sum_{l=0}^{2^{j_{1}-1}}\left(G_{j_{1}+1}^{\text {per }}\right)^{*}(k, l) U \psi_{j_{1}, l}^{\text {per }}=U \phi_{j_{1}+1, k}^{\text {per }} .
\end{aligned}
$$

Repeating this reasoning it can be proven that for $j>j_{1}, \Phi_{j, k}=U \phi_{j, k}^{p e r}$.

Taking into account the previous theorem, we can consider unitary operators $U: L^{2}(\mathbb{T}) \rightarrow \mathcal{H}$ to construct MRAs in the separable Hilbert $\mathcal{H}$. Given $U$ we define $\Phi_{j, k}$ and $\Psi_{j, k}$ by (8) and (9), respectively.

Remark 3.2: If several coefficients of an element of $\mathcal{H}$ in a wavelet-like basis are small, i.e., we have an effectively sparse representation, we say that the basis compresses these coefficients. Given $f \in \mathcal{H}$, since $\left\langle f, \Phi_{j, k}\right\rangle_{\mathcal{H}}=\left\langle U^{*} f, \phi_{j, k}^{\text {per }}\right\rangle_{L^{2}(\mathbb{T})}$ and $\left\langle f, \Psi_{j, k}\right\rangle_{\mathcal{H}}=\left\langle U^{*} f, \psi_{j, k}^{\text {per }}\right\rangle_{L^{2}(\mathbb{T})}$, then the compression of the coefficients of $f$ depends on the compression of the coefficients of $U^{*} f$.

Remark 3.3: For $f \in \mathcal{H}$, let $P_{n}(f)$ be the projection of $f$ onto $V_{n}$. We have

$$
P_{n}(f)=\sum_{k=0}^{2^{j_{0-1}}}\left\langle f, \Phi_{j_{0}, k}\right\rangle_{\mathcal{H}} \Phi_{j_{0}, k}+\sum_{j=j_{0}}^{n} \sum_{k=0}^{2^{j^{-}}-1}\left\langle f, \Psi_{j, k}\right\rangle_{\mathcal{H}} \Psi_{j, k} .
$$

Since $U$ is unitary,

$$
\left\|f-P_{n}(f)\right\|_{\mathcal{H}}^{2}=\left\|U^{*} f-U^{*} P_{n}(f)\right\|_{L^{2}(\mathrm{~T})}^{2} .
$$

Thus, the rate of approximation of $P_{n}(f)$ to $f$ in the norm of $\mathcal{H}$ is the same as the rate of approximation of $U^{*} P_{n} f$ to $U^{*} f$ in the norm of $L^{2}(T)$. Note that

$$
U^{*} P_{n}(f)=\sum_{k=0}^{2^{j_{0}-1}}\left\langle U^{*} f, \phi_{j_{0}, k}^{\text {per }}\right\rangle_{L^{2}(\mathrm{~T})} \phi_{j_{0}, k}^{\text {per }}+\sum_{j=j_{0}}^{n} \sum_{k=0}^{2^{j}-1}\left\langle U^{*} f, \psi_{j, k}^{\text {per }}\right\rangle_{L^{2}(\mathrm{~T})} \psi_{j, k}^{\text {per }}
$$

is the projection of $U^{*} f$ onto $V_{n}^{p e r}$.

\section{B. Construction of a MRA for $\mathcal{H}$ starting from an orthonormal basis of $\mathcal{H}$, filter pairs, and a unitary matrix}

Theorem 3.4: Let $\left\{e_{n}\right\}_{n=0}^{\infty}$ be an orthonormal basis of $\mathcal{H}$ and $\left(H_{j}, G_{j}\right), j \geq j_{0}+1$ be filter pairs. Then $\cup_{j=j_{0}}^{\infty}\left\{\Phi_{j, k}\right\}_{k=0}^{2^{j_{0-1}}}$ is a scaling-like system for $\mathcal{H}$ with a wavelet-like system $\cup_{j=j_{0}}^{\infty}\left\{\Psi_{j, k}\right\}_{k=0}^{j^{j}-1}$ and filter pairs $\left(H_{j}, G_{j}\right), j \geq j_{0}+1$, if and only if there exist $j_{1} \geq j_{0}$ and a unitary matrix

$$
T=\left(\begin{array}{c}
A_{j_{1}} \\
B_{j_{1}} \\
B_{j_{1}+1} \\
\vdots
\end{array}\right),
$$

i.e., its rows and columns form orthonormal systems in $\ell^{2}(Z)$, such that

$$
\left(\Phi_{j_{1}}\right):=A_{j_{1}}(u),
$$




$$
\begin{gathered}
\left(\Psi_{j}\right):=B_{j}(u), \quad j \geq j_{1}, \\
\left(\Phi_{j-1}\right)=H_{j}\left(\Phi_{j}\right), \quad j_{0}+1 \leq j \leq j_{1}, \\
\left(\Psi_{j-1}\right)=G_{j}\left(\Phi_{j}\right), \quad j_{0}+1 \leq j \leq j_{1},
\end{gathered}
$$

and

$$
\left(\Phi_{j}\right)=H_{j}^{*}\left(\Phi_{j-1}\right)+G_{j}^{*}\left(\Psi_{j-1}\right), \quad j>j_{1} .
$$

Proof: With $\cup_{j=j_{0}}^{\infty} V_{j}$ the matrix with entries $\left\langle\Phi_{j_{1}, k}, e_{l}\right\rangle_{\mathcal{H}}, k=0, \ldots, 2^{j_{1}}-1, l \geq 0$ and $B_{j}$ the matrix with entries $\left\langle\Psi_{j, k}, e_{l}\right\rangle_{\mathcal{H}}, k=0, \ldots, 2^{j}-1, l \geq 0, j \geq j_{1}$, the proof follows immediately from Theorem 2.9.

Remark 3.5: If

$$
\begin{gathered}
M_{j, j^{\prime}}=\left(\prod_{s=j+1}^{j^{\prime}} H_{s}\right) A_{j^{\prime}}, \quad j \leq j^{\prime}, \\
N_{j, j^{\prime}}=\left(\prod_{s=j+1}^{j^{\prime}} G_{s}\right) A_{j^{\prime}}, \quad j \leq j^{\prime}, \\
M_{j, j^{\prime}}=\left(\prod_{s=j^{\prime}+1}^{j} H_{j+j^{\prime}+1-s}^{*}\right) A_{j^{\prime}}, \quad j \geq j^{\prime},
\end{gathered}
$$

and

$$
N_{j, j^{\prime}}=\left(\prod_{s=j^{\prime}+2}^{j} H_{j+j^{\prime}+2-s}^{*}\right) G_{j^{\prime}+1}^{*} B_{j^{\prime}}, \quad j>j^{\prime},
$$

then

$$
\begin{gathered}
\left(\Phi_{j}\right):=M_{j, j_{1}}(u), \quad j_{0} \leq j \leq j_{1}, \\
\left(\Psi_{j}\right):=N_{j, j_{1}}(u), \quad j_{0} \leq j<j_{1}, \\
\left(\Phi_{j}\right):=M_{j, j_{1}}(u)+\sum_{j^{\prime}=j_{1}}^{j-1} N_{j, j^{\prime}}(u), \quad j>j_{1},
\end{gathered}
$$

and

$$
\left(\Psi_{j}\right):=B_{j}(u), j \geq j_{1}
$$

\section{A hybrid procedure}

We have presented above two ways of defining a MRA on $\mathcal{H}$.

(1) Take a unitary operator $U: L^{2}(\mathbb{T}) \rightarrow \mathcal{H}$ and define the scaling-like vectors $\Phi_{j, k}$ and waveletlike vectors $\Psi_{j, k}$ in $H$ as the image under $U$ of the scaling functions $\phi_{j, k}^{\text {per }}$ and wavelets $\psi_{j, k}^{p e r}$, respectively. This method is described in Theorem 3.1. Moreover, by this theorem, this method is the only way to obtain a MRA in $\mathcal{H}$ with filters $H_{j}^{\text {per }}$ and $G_{j}^{\text {per }}$. 
(2) Take an orthonormal basis $\left\{e_{n}\right\}$ of $\mathcal{H}$, filters $H_{j}, G_{j}$ and a unitary matrix $T$ and define the scaling-like vectors $\Phi_{j, k}$ and wavelet-like vectors $\Psi_{j, k}$ in $\mathcal{H}$ using relations (11)-(15). This method is described in Theorem 3.4.

In the first approach we work with known filters that have good properties. For example, the compression action of any wavelet (or wavelet-like) basis depends on the filters (see, e.g., Ref. 12). Moreover, as was noted in Remarks 3.2 and 3.3, the obtained MRA in $\mathcal{H}$ and the periodic MRA in $L^{2}(\mathbb{T})$ have the same rate of convergence of the projections and the same compression properties.

With the second approach we can obtain scaling-like and wavelet-like vectors in $\mathcal{H}$ that special properties. If each row of $T$ has only a finite number of non-null entries, i.e., $\Phi_{j, k}$ and $\Psi_{j, k}$ are finite linear combinations of the elements of the basis $\left\{e_{n}\right\}_{n=0}^{\infty}$ and each $e_{n}$ is, for example, $C^{\infty}$ or a polynomial, $\Phi_{j, k}$ and $\Psi_{j, k}$ will also be $C^{\infty}$ or a polynomial. Moreover, if each $e_{n}$ has an explicit expression $\Phi_{j, k}$ and $\Psi_{j, k}$ will also have an explicit expression.

For taking the advantages of both approaches, we can combine them considering in the second procedure the filters $H_{j}^{p e r}, G_{j}^{p e r}$ and a unitary matrix $T$ with only a finite number of non-null entries in each row. Given $0 \leq j_{0}<j_{1}$, a concrete and simple case is to consider $V_{j}=\operatorname{span}\left\{e_{n}\right\}_{n=0}^{2^{j}-1}$ and $W_{j}$ $=\operatorname{span}\left\{e_{n}\right\}_{n=2 j}^{j+1}$ for $j \geq j_{1}$. Now, given the filters $\left(H_{j}^{\text {per }}, G_{j}^{\text {per }}\right)$, we define $\left(\Phi_{j-1}\right),\left(\Psi_{j-1}\right), j_{0}+1 \leq j$ $\leq j_{1}$, using (13) and (14), respectively. The projection onto $V_{j_{1}}$ of a function can have a dense representation in the original basis $\left\{e_{n}\right\}_{n=0}^{j_{1}-1}$ but can be effectively sparse in the new basis $\left\{\Phi_{j_{0}, k}\right\}_{k=0}^{j_{0}^{j_{0}}} \cup \cup_{j=j_{0}}^{j_{1}-1}\left\{\Psi_{j, k}\right\}_{k=0}^{2^{j}-1}$. In Sec. IV we present wavelet-like bases constructed in this manner. It is important to note that the coefficients corresponding to the new basis can be obtained from those corresponding to the original basis using (2).

\section{SOME EXAMPLES}

In the following examples the filters $\left(H_{j}^{p e r}, G_{j}^{\text {per }}\right)$ correspond to the periodic Daubechies scaling and wavelet functions with 6 vanishing moments.

\section{A. Orthonormal trigonometric wavelet-like bases}

Given $0 \leq j_{0} \leq j_{1}$, for $j \geq j_{1}$, we consider in $L^{2}(T)$ the spaces

$$
V_{j+1}=\operatorname{span}\left\{1, \cos x, \ldots, \cos \left(2^{j}-1\right) x, \sin x, \ldots, \sin 2^{j} x\right\}
$$

and

$$
W_{j+1}=\operatorname{span}\left\{\cos 2^{j} x, \ldots, \cos \left(2^{j+1}-1\right) x, \sin \left(2^{j}+1\right) x, \ldots, \sin 2^{j+1} x\right\} .
$$

These spaces are considered in Ref. 4. Considering $j_{0}=2$ and $j_{1}=7$, Fig. 1 shows the graphs of some of these scaling-like and wavelet-like functions. Figure 2 shows the pattern and the number $n z$ of coefficients in the representation of some functions in this basis that are greater than $10^{-7}$ in absolute value. Considering also the number $n z_{o}$ of coefficients in the representation of the functions in the original basis that are greater than $10^{-7}$ in absolute value, the compression ratios $c$ $=n z_{o} / 256$ and $c n=n z / 256$ are also included. For the last function the roles of $H_{j}$ and $G_{j}$ were interchanged to obtain a greater compression. In all cases the coefficients in the original basis were sorted in ascending order to improve the compression action of $H_{j}$ and $G_{j}$.

\section{B. Orthonormal wavelet-like bases based on eigenfunctions}

Let $\mu$ be a (Borel) measure on the interval $J$ and consider the Hilbert space $\mathcal{H}=L^{2}(\mu ; J)$. Let $\left\{e_{n}\right\}_{n=0}^{\infty}$ be an orthonormal basis of $L^{2}(\mu ; J)$. Let $L$ be a self-adjoint positive differential operator defined on the set $C^{\infty}(J)$. Suppose that the elements of the basis $\left\{e_{n}\right\}_{n=0}^{\infty} \subset C^{\infty}(J)$ are eigenfunctions of $L$ with positive eigenvalues $\lambda_{n}$, i.e., $L e_{n}=\lambda_{n} e_{n}$. Suppose now that $S$ is a self-adjoint positive differential operator, such that $\langle S g, g\rangle_{L^{2}(\mu ; J)} \approx\langle L g, g\rangle_{L^{2}(\mu ; J)}, g \in C^{\infty}(J)$. Consider the equation 

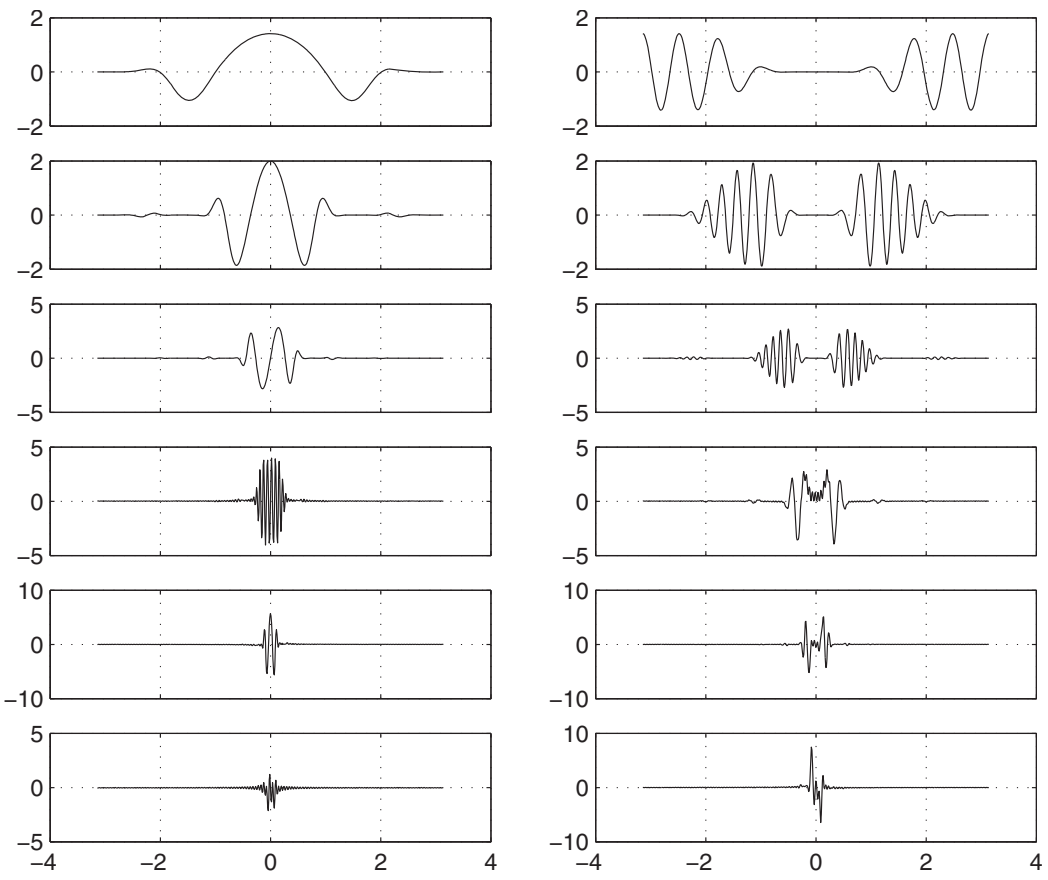

FIG. 1. Orthonormal trigonometric scaling-like functions (left column) and wavelet-like functions (right column) for $\mathrm{j}$ $=7, \ldots, 2$ with $\mathrm{k}=1,1,17,13,1,2$, respectively.

$$
S u=f,
$$

with $f \in L^{2}(\mu ; J)$. A Galerkin scheme for Eq. (24) is to consider some $j_{1}>j_{0} \geq 0$ and to find $v$ $\in V_{j_{1}}$, such that

$$
\langle S v, w\rangle_{L^{2}(\mu ; J)}=\langle f, w\rangle_{L^{2}(\mu ; J)}, \quad \forall w \in V_{j_{1}}
$$

The Galerkin scheme converges in the norm $\|g\|_{L}=\langle L g, g\rangle_{L^{2}(\mu ; J)}$. Noting that $V_{j_{1}}=\operatorname{span}\left\{e_{n}\right\}_{n=0}^{2^{j_{1}}-1}$, solving (25) is equivalent to finding a $2^{j_{1}}$-dimensional vector $d$, such that

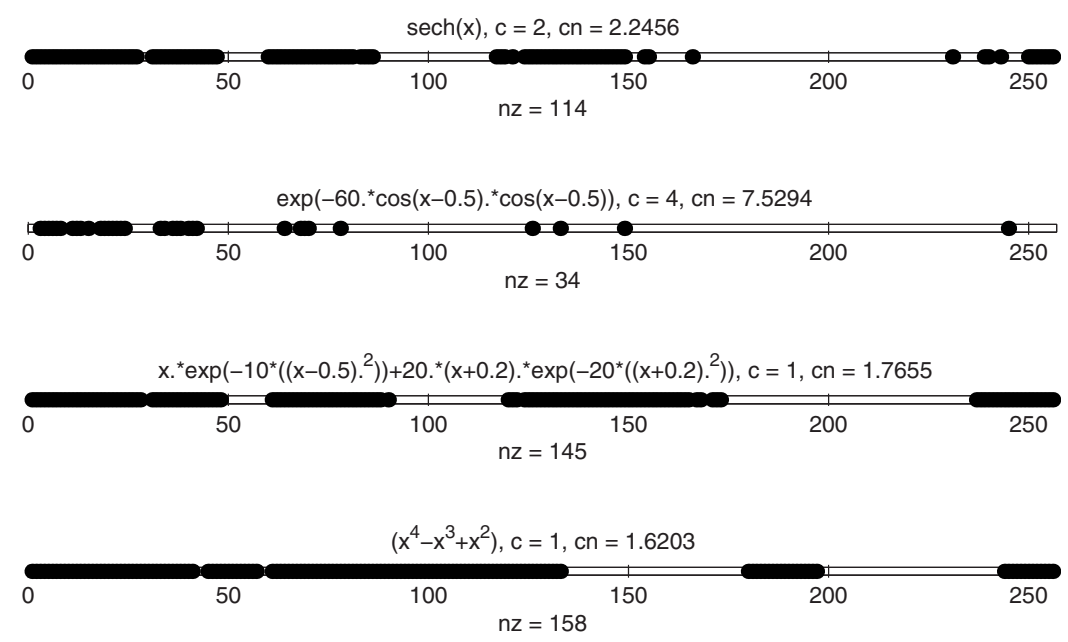

FIG. 2. Pattern and number $n z$ of coefficients in the representation of some functions in the orthonormal trigonometric wavelet-like bases that are greater than $10^{-7}$ in absolute value, along with the compression coefficients $c$ and $c n$. For the last function the roles of $H_{j}$ and $G_{j}$ were interchanged to improve the compression. 


$$
S_{2 j_{1}} d=s_{2 j_{1}}
$$

where $S_{2 j_{1}}$ is the matrix with entries $\left\langle S e_{m}, e_{n}\right\rangle_{L^{2}(\mu ; J)}$ and $s_{2 j_{1}}$ is the vector with entries $\left\langle f, e_{n}\right\rangle_{L^{2}(\mu ; J)}$. Consider the diagonal matrix $D_{2 j_{1}}$ with diagonal entries equal to $\lambda_{m}^{1 / 2}, m=0, \ldots, 2^{j_{1}}-1$. (26) can be rewritten as

$$
M_{2 j_{1}} x=b_{2 j_{1}},
$$

where $M_{2 j_{1}}=D_{2 j_{1}}^{-1} S_{2 j_{1}} D_{2 j_{1}}^{-1}, x=D_{2 j_{1}} d$, and $b_{2 j_{1}}=D_{2 j_{1}}^{-1} s_{2 j_{1}}$. Let $g=\sum_{n=0}^{2^{j_{1}}-1} \lambda_{n}^{-1 / 2} x_{n} e_{n}$. Since $\left\langle M_{2 j_{1}} x, x\right\rangle$ $=\langle S g, g\rangle_{L^{2}(\mu ; J)} \approx\langle L g, g\rangle_{L^{2}(\mu ; J)}=\|x\|^{2}$, then the ratios of the largest and smallest eigenvalues of the matrices $M_{2} j_{1}$ are uniformly bounded, i.e., are independent of $j_{1}$. So, the condition numbers of the matrices $M_{2 j_{1}}$ are uniformly bounded. Since the transformation from the basis $\left\{e_{n}\right\}_{n=0}^{2^{j_{1}}-1}$ to the new basis $\left\{\Phi_{j_{0}, k}\right\}_{k=0}^{2^{j_{0-1}}} \cup \cup_{j=j_{0}}^{j_{1}-1}\left\{\Psi_{j, k}\right\}_{k=0}^{2^{j}-1}$ is orthogonal, in this new basis the conditions numbers are uniformly bounded too.

Next we apply the previous analysis to perturbed Schrödinger and Chebyshev differential equations. These equations were considered in Refs. 8 and 9, respectively (see Remark 4.3 below).

Example 4.1: Let $J=(-\infty, \infty), \mu \equiv 1$, and $e_{n}$ be the Hermite functions, i.e., $e_{n}(x)$ $=h_{n}(x) e^{-x^{2} / 2} / \sqrt{2^{n} n ! \sqrt{\pi}}$, where $h_{n}$ are the Hermite polynomials. These $e_{n}$ are eigenfunctions of the Schrödinger operator $-d^{2} / d x+x^{2} I$. Let $a(x), b(x)$ be measurable functions, such that $0<a_{1}$ $\leq a(x) \leq a_{2}, 0<b_{1} / x^{2} \leq b(x) \leq b_{2} / x^{2}$ for some positive constants $a_{1}, a_{2}, b_{1}, b_{2}$. Then, by the previous discussion, the resultant algebraic system for the Galerkin scheme of the perturbed Schrödinger equation,

$$
-\left(a(x) u^{\prime}(x)\right)^{\prime}+b(x) u(x)=f(x)
$$

can be preconditioned by a diagonal matrix so that the condition number is uniformly bounded. Equation (28) can be viewed as a model of second-order elliptic operators with unbounded coefficients.

Example 4.2: A similar result can be obtained for the degenerate elliptic equation,

$$
-\left(1-x^{2}\right)^{1 / 2} \frac{d}{d x}\left(a(x) \frac{d u}{d x}\right)=f(x),
$$

with $\underline{0} \leq a_{1}\left(1-x^{2}\right)^{1 / 2} \leq a(x) \leq a_{2}\left(1-x^{2}\right)^{1 / 2}$, if we consider $J=[-1,1], \mu=\left(1-x^{2}\right)^{-1 / 2} d x, e_{0}(x)$ $=1 / \sqrt{\pi}, e_{n}(x)=\sqrt{2 / \pi} T_{n}(x), n=1,2, \ldots$, where $T_{n}(x)=\cos (n \operatorname{arc} \cos t)$ are the Chebyshev polynomials. The $e_{n}$ are eigenfunctions of the Chebyshev operator $-\left(1-x^{2}\right)^{1 / 2} d / d x\left(\left(1-x^{2}\right)^{1 / 2} d / d x\right)$.

The graphs of some of the scaling-like and wavelet-like functions considered in Example 4.1 with $j_{0}=2$ and $j_{1}=6$, and in Example 4.2 with $j_{0}=2$ and $j_{1}=8$, appear in Figs. 3 and 4 , respectively.

Remark 4.3: The eigenfunctions in the previous examples are considered in the articles ${ }^{8,9}$ for constructing orthonormal wavelet-like bases using a different approach. Nevertheless, if we consider the Meyer scaling function $\phi$ and wavelet $\psi$, and $j_{1} \geq j_{0}=0$, these scaling-like and waveletlike functions can be obtained as in Theorem 3.4 with the filter pairs of $\phi_{j, k}^{\text {per }}, \psi_{j, k}^{\text {per }}$, and $T(k, \sigma(n))=\widehat{\phi_{j_{1}, k}}(2 \pi n)$ for $k=0, \ldots, 2^{j_{1}}-1, \quad|n|<\frac{2}{3} 2^{j_{1}}, T\left(2^{j}+k, \sigma(n)\right)=\widehat{\psi_{j, k}}(2 \pi n)$ for $j \geq j_{1}, k$ $=0, \ldots, 2^{j}-1, \frac{1}{3} 2^{j}<|n|<\frac{4}{3} 2^{j}$, where $\sigma: \mathbb{Z} \rightarrow \mathbb{Z}^{+}$is given by $\sigma(n)=2 n$ if $n \geq 0$ and $\sigma(n)=2|n|-1$ if $n<0$. The resultant algebraic systems for Eqs. (28) and (29) corresponding to the Galerkin scheme in these wavelet-like bases are also considered. It is proved that they can be preconditioned by a diagonal matrix and that the scheme converges in the $L^{2}(\mu ; J)$ norm. A periodic pseudodifferential operator connected with the equation $S u=f$ is introduced. 

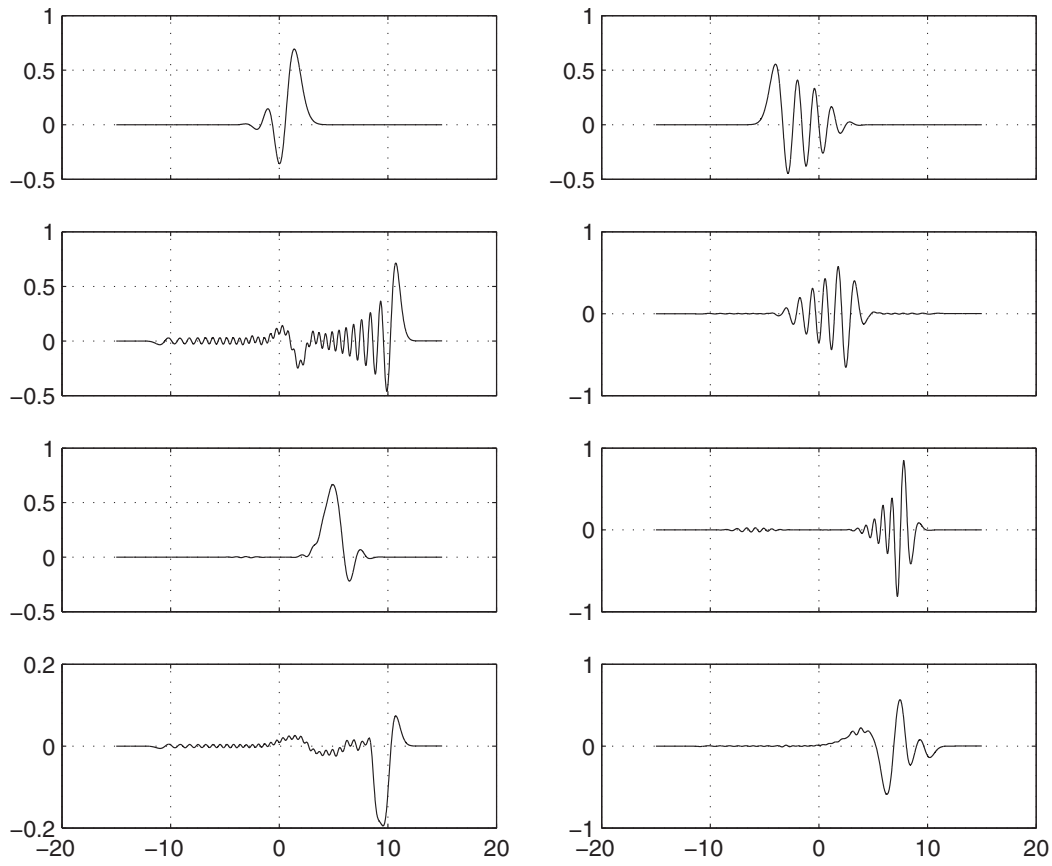

FIG. 3. Orthonormal wavelet-like bases based on Hermite polynomials. Scaling-like functions (left column) and waveletlike functions (right column) for $\mathrm{j}=5,4,3,2$ with $\mathrm{k}=1,15,1,1$, respectively.

\section{FINAL REMARKS}

We have presented a general method for constructing wavelet-like bases in a Hilbert space that can lead to smooth wavelet-like functions with explicit expressions that permit effectively
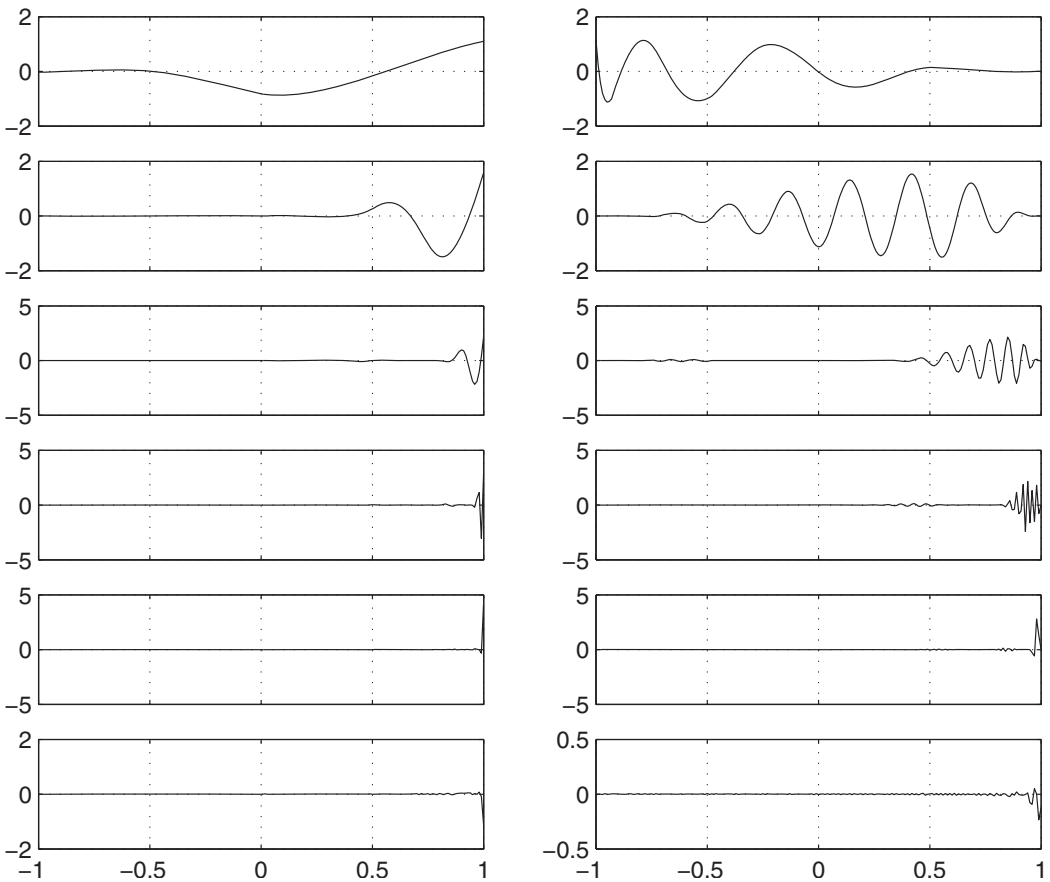

FIG. 4. Orthonormal wavelet-like bases based on Chebyshev polynomials. Scaling-like functions (left column) and wavelet-like functions (right column) for $\mathrm{j}=7, \ldots, 2$ with $\mathrm{k}=1$, respectively. 
sparse representations. The method was illustrated in a particular simple case using trigonometric functions and eigenfunctions of differential operators. Future investigations can explore other implementations of the method and other special functions. In particular, if the $e_{n}$ are multivariate functions or are defined on certain domain, then we can obtain wavelet-like functions with this characteristic. Also, different filter pairs and matrices $T$ can be used.

\section{ACKNOWLEDGMENTS}

Research partially supported by ANPCyT (Grant No. PICT-2007-00865, Foncyt), Universidad Nacional de San Luis (Grant No. P-317902), and Consejo Nacional de Investigaciones y Técnicas (Grant No. PIP 112-200801-01070), Argentina.

${ }^{1}$ Beylkin, G., Coifman, R., and Rokhlin, V., "Fast wavelet transforms and numerical algorithms I," Commun. Pure Appl. Math. 44, 141 (1991).

${ }^{2}$ Beylkin, G., Coifman, R., and Rokhlin, V., in Wavelets and Their Applications, edited by Ruskai, M. B., Beylkin, G., Coifman, R., Daubechies, I., Mallat, S., Meyer, Y., and Raphael, L. (Jones and Bartlett Books in Mathematics, Boston, 1992), pp. 181-210.

${ }^{3}$ Chui, C. K., An Introduction to Wavelets (Academic Press, New York, 1992).

${ }^{4}$ Chui, C. K. and Mhaskar, H. N., "On trigonometric wavelets," Constructive Approx. 9, 167 (1993).

${ }^{5}$ Dahmen, W., "Wavelet and multiscale methods for operator equations," Acta Numerica 6, 55 (1997)

${ }^{6}$ Dahmen, W., "Wavelets methods for PDEs—some recent results," J. Comput. Appl. Math. 128, 133 (2001).

${ }^{7}$ Dai, D. Q., "Wavelets and orthonormal polynomial based on harmonic oscillator eigenstates," J. Math. Phys. 41, 3086 (2000).

${ }^{8}$ Dai, D. Q., Han, B., and Jia, R. Q., “Galerkin analysis for Schrödinger equation by wavelets,” J. Math. Phys. 45, 855 (2004).

${ }^{9}$ Dai, D. Q. and Lin, W., "Orthonormal polynomial wavelets on the interval," Proc. Am. Math. Soc. 134, 1383 (2006).

${ }^{10}$ Daubechies, I., Ten Lectures on Wavelets (Society for Industrial and Applied Mathematics, Philadelphia, PA, 1992).

${ }^{11}$ Fang, L. and Thews, R. L., Wavelets in Physics (World Scientific, Singapore, 1998).

${ }^{12}$ Ford, J., "Wavelet-based preconditioning of dense linear systems," Ph.D. thesis, University of Liverpool, 2001.

${ }^{13}$ Hernández, E. and Weiss, G., A First Course on Wavelets (CRC, Boca Raton, FL, 1996).

${ }^{14}$ Meyer, Y., Ondelettes et Opéreteurs I. Ondelettes (Herman, Paris, 1990).

${ }^{15}$ van den Berg, J. C., Wavelets in Physics (Cambridge University Press, Cambridge, 2004).

${ }^{16}$ Wojtaszczyk, P., A Mathematical Introduction to Wavelets (Cambridge University Press, Cambridge, 1997). 